\title{
The Application of the Internet of Things in Healthcare
}

\author{
Fayez Hussain Alqahtani \\ King Saud University \\ P. O. BOX 2454, Riyadh 11451 \\ Kingdom of Saudi Arabia
}

\begin{abstract}
Communication and sensing devices, as well as their respective software, have become versatile for Information Technology (IT) solutions in different contexts. The emergent technology known as the Internet of Things (IoT) has helped both practitioners and researchers to design innovative solutions in healthcare. The IoT-enabled healthcare research is significant due to its valuable implications, including higher quality and lower cost of services and reliable preventive care. This paper reviews the current literature on the IoT in healthcare and discusses its applications and enabling technologies, as well as critical challenges.
\end{abstract}

\section{General Terms}

Healthcare Information Systems, Internet of Things

\section{Keywords}

Internet of Things; IoT; Healthcare; Information Systems, Healthcare Information Systems

\section{INTRODUCTION}

The advancement in information and communication technologies has opened the door for innovations in many aspects of daily life. The emergent technology known as the Internet of Things (IoT) has helped both practitioners and researchers to design innovative solutions in different contexts, specifically in healthcare [1]. The application of IoT devices is enormous in healthcare, estimated as accounting for over $30 \%$ of its application in all fields [2].

The rapid advancement of cloud computing, mobile applications and wearable devices facilitates the IoT's role in transforming the traditional approach to healthcare into smart and personalised healthcare. The IoT-enabled healthcare systems monitor several medical parameters, such as blood pressure (BP) and glucose levels, as well as body temperature, using smart sensors, computer networks and a remote server [3]. These healthcare systems could also provide basic suggestions for treatment, which are very helpful, particularly when applied in homes or communities [1].

As noted by [4], the IoT-enabled healthcare research is significant due to its valuable implications, including higher quality and lower cost of services and reliable preventive care. According to [5], IoT-based healthcare is an active interdisciplinary research area where different methodologies, ranging from those of engineering and computer science to that of behavioural science, are deployed to design innovative IT solutions to practical medical issues. Further research is needed to provide an overview of the IoT, its architectures and various technologies in the healthcare context [6]. Therefore, this paper aims to review the current literature on IoT-enabled healthcare. It illustrates the application of the IoT in healthcare, as well as its enabling technologies and critical challenges.

\section{ORIGIN OF IOT}

The term Internet of Things was first coined by [7], the founder of the Auto-ID centre at the Massachusetts Institute of Technology. Auto-ID is used to describe all sorts of measures to identify and improve applications, such as work automation, efficiency enhancement, error reduction and so on. In 2003, the Auto-ID centre released the electronic product code (EPC) network. The EPC enables tracking objects moving from one location to another. This gives an idea for the IoT implementation, where microchips can be used to create a network for mainstream commercial means [4]. The radio frequency identification (RFID) implementation further cements the opportunities for developing the IoT as a new IT paradigm in both academic and industrial environments [8].

In the 2005 report of the International Communication Union, the IoT was proposed as a collaboration of computing and sensor-based technologies, such as sensors, wireless networks, embedded systems, object identifiers and nanotechnologies. This combination enables the objects to be tagged, sensed and controlled over the networks. The IoT is considered a combination of technologies aimed to provide interaction and communication among linked devices [8]. Several enterprise systems, such as healthcare, the industrial sector and so on, based on IoT applications have also been developed [9]. Developing countries also seem to be keenly interested in the IoT. The former Chinese President inaugurated a national IOT research centre in 2009 and delivered a speech about the need for advanced research and development in the IoT field [10].

\section{APPLICATIONS OF IOT}

The IoT has a wide range of applications and can be successfully implemented in areas such as the healthcare sector, retail business, travel and tourism, hypermarkets, event management, the manufacturing sector, environmental systems, logistic systems, hotels and restaurants and a lot more. This broad spectrum of application areas depicts the IoT as playing a vital role in the smooth functioning of society.

\subsection{Industrial Applications of IoT}

The use of the IoT in the business sector can sharply enhance the day-to-day operations by integrating real-time data processing with the utilisation of resources, such as critical data storage, online payment and aggregated Quality of Service (QoS) [11]. This will make the organisations' business models more profit oriented, develop competitive products and provide real-time information processing with optimised resource utilisation. This approach is profitable for manufacturers and their business partners as they can integrate the enterprises' resources for optimum utilisation. Presently, many products being manufactured use unique identifiers such as RFID, barcodes, Quick Response (QR) codes, 
intelligent sensors and so on. Such identifiers help locate the goods during their life cycles.

\subsection{Healthcare Applications of IoT}

A vital application area of the IoT is the healthcare sector. The IoT has played a crucial role in this field by enhancing service quality while reducing costs [12]. It is possible to track health parameters, such as BP, blood glucose, body temperature and so on, in real time by using wireless sensors. The development of improved sensors, better data processing technologies and advanced technologies for wireless communication has led to the increasing implementation of the IoT in the healthcare sector. The development of wearable body sensor networks (WBSNs) to continuously monitor patients' activities is another milestone for the implementation of the IoT.

\section{IOT IN HEALTHCARE}

Medical devices have undergone drastic changes, from the traditional unconnected equipment to wirelessly reprogrammable devices. These advancements include the emergence of medical IoT systems that can even be connected to cell phones. The medical IoT is basically a system comprising mainly health-monitoring devices. Patients' health parameters are remotely recorded by a back-end system. Afterwards, the back- end system analyses the recorded data and provides appropriate feedback to the clinical staff. The feedback helps specialists determine the current health situation of patients and immediately react to critical cases [13].

A medical device can be used to monitor health parameters. Alternative devices, such as a smart watch or a mobile phone, may be good substitutes on the move [12]. At the same time, it should be taken into consideration that the dataset recorded by these devices is of utmost importance as it comprises the health records of patients. This system is quite useful for healthcare clinics, hospitals or outpatient clinics. The medical IoT system is a sophisticated setup that contains a variety of mechanisms and systems, such as medical equipment, smart sensors, network gateways, cloud computing, big data, clinical information systems and so on, that cooperate to control the healthcare environment.

\section{ENABLING TECHNOLOGIES OF IOT IN HEALTHCARE}

Currently, communication and sensing devices and their respective software have become versatile for healthcare-IoT systems [14]. A wide range of good decisions made by these systems is driven by the use of the following IoT-enabling technologies.

\subsection{Identification Technology}

A working model of the IoT may have a large number of nodes. An authorised node may generate data and could have access to data, irrespective of its location. To achieve this goal, it becomes necessary to efficiently seek and identify the nodes. The identification process assigns a unique identification number (UID) to each node in order to provide unambiguous information exchange via the node. Each individual resource in a system is given a digital UID. For example, in a hospital, a doctor, a nurse, or any other staff member is provided with a UID. This helps create relations among different entities in the digital domain. It enables the prompt location of the available objects in the network without fail. Various standards have been proposed for the unique identification of objects in the digital domain, such as a universally unique identifier (UUID). One such standard has been developed by the Open Software Foundation.

\subsection{Communication Technologies}

While considering an IoT-based system, the communication technologies enable the network infrastructure. IoT networks are based on heterogeneous frequencies, standards and transmission rates for transferring data. These networks can be further be classified as long-distance and short-distance technologies. Long-distance technologies are intended to deal with regular means of communication, such as the internet or mobile phones. Short-distance communication mostly utilises wireless technologies, such as Bluetooth, Infrared Data Association (IrDA), Wi-Fi, ultra-wideband (UWB), RFID and so on. All these technologies enable data transmission over a short distance. The features of these technologies may differ in terms of the installation cost, transmission rates, distance, the number of entities, power consumption, maintenance cost and so on, based on differences in working radio frequencies and security standards.

\subsection{Location Technology}

In modern tracking systems, real-time location systems (RTLS) help locate objects. The global positioning system (GPS) is considered the most important RTLS. This satellitedependent navigation system is capable of locating objects under various weather conditions. For healthcare applications, the GPS can help in precisely locating ambulances, patients, doctors and so on. However, these navigation systems, such as the GPS or China's Beidou system (BDS), are ineffective indoors due to the construction structure that hampers the satellites' transmission signals. Hence, it becomes necessary to develop a local positioning system (LPS) that can replace the GPS and work with better accuracy indoors. An LPS measures the radio signals that travel between an object and an array of receivers aimed to locate it [15]. There is a great possibility to create smart indoor positioning network systems by combining the GPS or the BDS with an LPS in a high bandwidth wireless communication network.

\subsection{Sensing Technologies}

Sensors form the heart of the IoT-based systems, as they are on-the-ground devices performing the critical part of monitoring processes, taking measurements and collecting data. With advancements in sensing technologies, it is possible to obtain continuous data from objects or even from living creatures, such as humans. For example, the pulse oximeter that was invented in the 1970s is used as a major device for diagnosis [16]. It helps a physician monitor a patient's heart rate (HR) and blood oxygen saturation ( $\mathrm{SpO} 2)$, which are critical for emergency services. Other types of sensors include temperature, pressure, water quality and smoke sensors. The instrument that analyses motion is a complicated device that comprises various sensors, including accelerometers, gyroscopes, surface electrodes, among others [16]. It is possible to transform all the received data from the sensors into a digital form and immediately transmit it over a network. The prevalence of wireless sensors has made it possible for people to wear portable sensors capable of automated data collection and transfer.

\subsection{Cloud Computing}

During its operations, an IoT system generates a huge amount of data that has to be stored, processed and shared [17]. Cloud computing forms the building block of the IoT architecture as it can support the storage and the processing of the immense data generated by individual sensors and devices. The cloud 
data centres collect data from the individual devices in an IoT system to analyse and share it with other sensor devices on the network. It is possible for the cloud data centres to enhance or decrease the computing capacity, depending on the demand.

Furthermore, research proves the huge potential of cloud computing for the next-generation smart systems for people with disabilities [17]. All the future IoT systems are supposed to be based on the cloud. The associated devices from the computers can be decoupled by cloud computing by avoiding individual installation [18]. An additional benefit of IoT devices is that they can be reconfigured without much user effort and time. Since cloud computing is reliable and platform independent, the setup of cloud centres in hospitals and medical centres facilitates resource sharing and leads to the development of highly reliable medical monitoring and management systems [19].

\section{EXAMPLES OF IOT SERVICES IN HEALTHCARE}

An IoT-based healthcare architecture has three main layers: information perception, network transmission and application service. The information perception layer primarily consists of sensors that are used to continuously monitor people's health statistics [20]. The collected data is transmitted over the networks and stored in cloud data centres. Wireless technologies, such as Wi-Fi, ZigBee, EnOcean and so on, are utilised to transmit data over the networks. The application service layer involves the IoT applied in a medical centre, providing a remote healthcare service [21].

\subsection{Glucose-Level Monitoring}

Many people are diabetic, and there is a need to continuously monitor their glucose levels. A medical IoT system is capable of continuously monitoring glucose levels in a non-invasive way. The patients use wearable sensors capable of tracking their health parameters, and the collected data is transferred via an internet protocol (IPV6) network to significant healthcare providers. The tracking device consists of a blood glucose collector, a mobile phone and an IoT-based medical acquisition detector to monitor the glucose level [25]. Glucose-level monitoring provides individual patterns of modifications in glucose levels that can be utilised to decide about meals, physical activities, medication times and so on.

\subsection{Electrocardiogram Monitoring}

In electrocardiogram (ECG) monitoring, the system keeps track of the HR and the basic rhythm, along with the identification of multifaceted arrhythmias, myocardial ischemia and prolonged QT intervals by recording the electrical activity of the heart. The ECG monitor consists of a wireless transmitter and a receiver. An automated application can identify an abnormal heart activity. The data is transferred in real time to mobile phones and the doctor's clinic via a network. The IoT system utilises algorithms for continuous ECG monitoring [20].

\subsection{Blood Pressure Monitoring}

A patient's BP can continuously be monitored by using a wearable sensor device. The machine consists of a BP apparatus with network-based communication abilities. Blipcare is such a device that uses a home Wi-Fi network to record $\mathrm{BP}$ and upload the recorded data. The device also has an LCD display to show the BP. A device for remotely monitoring BP has been proposed [21].

\subsection{Body Temperature Monitoring}

The change in body temperature is used to identify homeostasis, which forms an essential part of healthcare services. A TelosB mote, used in a medical IoT, has an embedded sensor to record body temperature. Jian and colleagues [22] propose a system that uses a home gateway for monitoring body temperature in a medical IoT. The home gateway uses infrared detection to transmit the recorded body temperature. The system primarily includes an RFID module that functions in collaboration with a body temperature monitoring device.

\subsection{Wheelchair Management}

Several studies have already been conducted to develop smart wheelchairs for people with disabilities who use the IoT application. For example, Yang [23] proposes an IoT-based healthcare system for persons with disabilities. The system utilises Wireless Body Area Networks WBANs technology to control and coordinate different sensors. The vibrations in the wheelchair are controlled by the system. It also keeps track of the status of the person using the wheelchair by monitoring his or her sitting position, as well as giving information about the surroundings.

\subsection{Heart Rate}

Smartphones are increasingly used as integral parts of a medical IoT. All the newer electronic devices are now controlled by smartphones. Several healthcare-related hardware products have been integrated into and a lot of software applications have been developed for smartphones [24]. Smartphones are currently equipped with a large number of healthcare applications. Several noncontact measuring sensors that work on image analysis algorithms are also used for healthcare applications. A typical smartphone is now capable of diagnosing diseases such as asthma, chronic obstructive pulmonary disease, cystic fibrosis, allergic rhinitis, nose-related symptoms of the respiratory tract, HR, $\mathrm{BP}, \mathrm{SpO} 2$, among others.

\section{CRITICAL ISSUES AND CHALLENGES OF IOT IN HEALTHCARE}

The IoT-based healthcare sector is experiencing tremendous growth. The use of IoT devices and sensors in the medical sector forms the basis of the e-health system. People use these devices to monitor their daily health statistics. Simultaneously, the devices use transmission networks to send/receive the health-related data of patients. This results in a potential threat by hackers. Hence, it becomes necessary to completely secure the IoT-based healthcare system. The medical IoT systems face the following major threats:

\subsection{Scalability}

Billions of interconnected IoT devices generate huge amounts of data for processing and storage [25]. The IoT system that handles these devices should be scalable. The large amount of data generated by the current system is stored by using big data over the cloud.

Interoperability: Several manufacturers provide different products, services and devices that are used in IoT systems. Unfortunately, these firms do not follow any standard protocol for the manufacturing and the use of these devices. This becomes a major cause of interoperability issues [24]. The existence of a large number of diverse devices and the management of value-added services are key standardisation issues at present. 


\subsection{Security}

Many people continuously wear medical sensor-based devices to keep track of their health statistics. In such scenarios, security becomes vital as any breach of it may prove to be critically life threatening [26]. Hence, the security of the information obtained by different sensors and devices in a medical IoT becomes indispensable. Proper policies and technical security measures are essential to enable data sharing among authorised users and organisations [27]. Different characteristics, such as confidentiality, integrity and availability of people's personal data, should be guaranteed in an IoT healthcare system. Efficient security of resources is another key requirement. The IoT-based healthcare systems should be equipped with foolproof mechanisms that utilise minimum resources with maximum security performance.

Physical security: The devices used in the IoT-based healthcare system should have tamper-resistant packaging [27]. It is possible for an attacker to take control of a device and alter it to obtain crucial data. Additionally, the routing algorithms used should be properly controlled to safeguard the transmitted data. The network nodes are always susceptible to attacks. Hence, there is a strong need for secure routing protocols in the transmission of data [28]. The IoT medical devices are equipped with procedures to access cloud services [26]. The services should be properly monitored so that patients' data can easily be tracked and controlled.

\subsection{Mobility}

It is a basic requirement for an IoT-based healthcare system to permit mobility of patients and devices so that the system is always functional, irrespective of the location. This feature makes it possible to connect heterogeneous patient environments [28].

Network type: Sometimes, the selection of a proper network becomes an issue. There are three main types of networks: data centric, service centric and patient centric. The datacentric network categorises the healthcare structure on the basis of captured health data [29]. The service-centric structure is based on the structures formed by the assembly of services provided by the system. The patient-centric structure is created by using the structure formed by the involvement of the people under treatment.

\section{DISCUSSION AND CONCLUSION}

The rapid advancement of cloud computing, mobile applications and wearable devices facilitates the IoT's role in transforming the traditional approach to healthcare into smart and personalised healthcare. The IoT-enabled healthcare systems can be categorised under three primary areas, as follows: monitoring and controlling, information sharing and collaboration, and big data and data analytics [11].

The IoT-enabled healthcare systems monitor several medical parameters, such as BP and glucose levels, as well as body temperature, by using smart sensors, computer networks and a remote server. Proper monitoring and control are indispensable aspects of a large collaborative network application. For example, in an IoT-based healthcare system, more than a dozen IoT devices from various external users interact via its applications. Although the monitoring and control devices work independently, they need to collaborate with one another in order to synchronise the received input. For a smart-building operation, several sensing devices in the building collaborate with one another [30].
There is an emerging trend of using smart healthcare systems to keep track of patients' medical information with small sensing devices, which collect data over a network in the course of the patients' daily routines [31]. For example, an IoT system that detects a person's fall can provide support to the elderly or people with disabilities who live independently. There is a need for continuous communication and interaction among various devices and humans for better collaboration in an IoT environment.

Another favourable area for IoT applications involves big data and data analytics [11]. The IoT systems produce huge amounts of data after a long time of usage [32]. This massive data can be kept in cloud-based data centres for processing [33]. A proper analysis of stored data in a cloud may reveal certain patterns based on which medical information analyst can predict the health risks for a person with disability and generate appropriate health alerts or basic suggestions for treatment, which are very helpful, particularly when applied in homes or communities.

Despite the value of implementing IoT solutions in healthcare, it might encounter some challenges, including security, scalability and mobility, yet security and privacy remain the most critical issues. The security of IoT-healthcare building blocks should be prioritised, including medical sensors, the network of IoT nodes and cloud services [34]. Furthermore, proper policies and technical security measures are essential to enable data sharing among authorised devices, users and organisations. Due to the potential risk of storing sensitive health data in IoT cloud services, further research should consider designing data-transparent cloud services. Researchers can employ a design science approach to develop IoT cloud services in a way that healthcare data can be traced and its usage can be controlled.

\section{REFERENCES}

[1] P. A. Laplante and N. Laplante, "The Internet of Things in healthcare: potential applications and challenges," IT Professional, vol. 18, pp. 2-4, May 2016.

[2] M. B. Blake, "An Internet of Things for healthcare," IEEE Internet Computing, vol. 19, pp. 4-6, August 2015.

[3] P. Po Yang, O. Amft, Y. Gao, and L. Xu, "Special issue on the Internet of Things (IoT): informatics methods for IoT-enabled health care," Journal of Biomedical Informatics, vol. 63, pp. 404-405, September 2016.

[4] Y. Yin, Y. Zeng, X. Chen, and Y. Fan, "The Internet of Things in healthcare: an overview," Journal of Industrial Information Integration, vol. 1, pp. 3-13, March 2016.

[5] P. L. L. Benny, I. Henry, and Y. Guang-Zhong, "Transforming health care: body sensor networks, wearables, and the Internet of Things," IEEE Pulse, vol. 7, pp. 4-8, January 2016.

[6] S. Madakam, R. Ramaswamy, and S. Tripathi, "Internet of Things (IoT): a literature review," Journal of Computer and Communications, vol. 3, pp. 164-173, May 2015.

[7] K. Ashton, "That 'Internet of Things' Thing," RFiD Journal, vol. 22, pp. 97-114, June 2009.

[8] N. Gershenfeld, R. Krikorian, and D. Cohen, "The Internet of Things," Sci. Am, vol. 291, pp. 76 - 81, Oct 2004.

[9] L. M. R. Tarouco, L. M. B., L. Z. Granville, L. M. R. Arbiza, F. Carbone, M. Marotta, and J. J. C. De Santanna, Internet of Things in healthcare: 
interoperability and security issues, IEEE International Conference Communications. Sydney, Australia: IEEE, 2012.

[10] F. Mattern and C. Floerkemeier, "From the Internet of Computers to the Internet of Things," in "From Active Data Management to Event-Based Systems", Vol. 6462, K. Sachs, I. Petrov \& P. Guerrero, Eds. Berlin, Heidelberg: Springer, 2010, pp. 242-259.

[11] I. Lee and K. Lee, "The Internet of things (IoT): applications, investments and challenges for enterprises," Business Horizons, vol. 58, pp. 431-440, August 2015.

[12] P. A. Williams and A. J. Woodward, "Cybersecurity vulnerabilities in medical devices: a complex environment and multifaceted problem," Medical Devices (Auckland), vol. 8, pp. 305-316, Julay 2015.

[13] D. Miorandi, S. Sicari, F. De Pellegrini, and I. Chlamtac, "Internet of Things: vision, applications and research challenges," Ad Hoc Networks, vol. 10, pp. 1497-1516, September 2012.

[14] M. Busch, C. Hochleitner, M. Lorenz, T. Schulz, M. Tscheligi, and E. Wittstock, "All in: targeting trustworthiness for special needs user groups in the Internet of Things," in Lecture Notes in Computer Science, M. Huth, N. Asokan, S. Čapkun, I. Flechais, and L. Coles-Kemp, Eds. Berlin: Springer, pp. 223-231, 2013.

[15] R. Peng and M. L. Sichitiu, Angle of arrival localization for wireless sensor networks. In Proceedings of the 3rd Annual IEEE Communications Society on Sensor and Ad Hoc Communications and Networks. Reston, VA, USA: IEEE, 2006

[16] K. K. Tremper and S. J. Barker, "Pulse oximetry," Anesthesiology, vol. 70, pp. 98-108, Jan 1989.

[17] J. Gubbi, R. Buyya, S. Marusic, and M. Palaniswami, "Internet of Things (IoT): a vision, architectural elements, and future directions," Future Generation Computer Systems, vol. 29, pp. 1645-1660, September 2013.

[18] D. Mulfari, A. Celesti, M. Fazio, M. Villari, and A. Puliafito, "Achieving assistive technology systems based on IoT devices in cloud computing," EAI Endorsed Transactions on Cloud Systems, vol. 1, pp. 1-6, February 2015.

[19] Y. Liu, B. Dong, B. Guo, J. Yang, and W. Peng, "Combination of cloud computing and Internet of Things (IOT) in medical monitoring systems," International Journal of Hybrid Information Technology, vol. 8, pp. 367-376, December 2015

[20] S. M. Riazul Islam, D. N. Kwak, M. H. Kabir, M. Hossain, and K.-S. Kwak, "The Internet of Things for health care: a comprehensive survey," IEEE Access, vol. 3, pp. $678-708$, June 2015.

[21] G. Zhang, C. Li, Y. Zhang, C. Xing, and J. Yang, "Seman medical: a kind of semantic medical monitoring system model based on the IoT sensors. In Proceedings of the 14th International Conference on e-Health Networking, Applications and Services (Healthcom 2012). Beijing, China: IEEE, 2012.
[22] Z. Jian, W. Zhanli, and M. Zhuang, "Temperature measurement system and method based on home gateway," CN 201110148247, 2012.

[23] L. Yang, Y. Ge, W. Li, W. Rao, and W. Shen, "A home mobile healthcare system for wheelchair users. In Proceedings of the 18th International Conference on Computer Supported Cooperative Work in Design. Hsinchu, Taiwan: IEEE, 2014.

[24] S. Sivagami, D. Revathy, and L. Nithyabharathi, "Smart health care system implemented using IoT," International Journal of Contemporary Research in Computer Science and Technology, vol. 2, February 2016.

[25] S. K. Datta, C. Bonnet, A. Gyrard, R. P. F. D. Costa, and K. Boudaoud, "Applying Internet of Things for personalized healthcare in smart homes. In Proceedings of the 24th Wireless and Optical Communication Conference. Taipei, Taiwan: IEEE, 2015.

[26] R. E. Samuel and D. Connolly, "Internet of Things-based health monitoring and management domain-specific architecture pattern," Issues in Information Systems, vol. 16, pp. 58-63, December 2015.

[27] A.-M. Rahmani, N. K. Thanigaivelan, T. N. Gia, J. Granados, B. Negas, P. Liljeberg, and H. Tenhunen, "Smart e-health gateway: bringing intelligence to Internet-of-Things-based ubiquitous healthcare systems. In Proceedings of the Annual IEEE Consumer Communications and Networking Conference. NV, USA: IEEE, January 2015.

[28] S. Agrawal and D. Vieira, "A survey on Internet of Things," Abakós, Belo Horizonte, vol. 1, pp. 78-95, May 2013.

[29] F. J. Riggins and S. F. Wamba, "Research directions on the adoption, usage, and impact of the Internet of Things through the use of big data analytics. In Proceedings of the 48th Hawaii International Conference on System Sciences. HI, USA, 2015.

[30] J. Yu, M. Kim, H. C. Bang, S. H. Bae, and S. J. Kim, "IoT as applications: cloud-based building management systems for the Internet of Things," Multimedia Tools and Applications, vol. 75, pp. 14583-14596, July 2015.

[31] T. Suzuki, H. Tanaka, S. Minami, H. Yamada, and T. Miyata, "Wearable wireless vital monitoring technology for smart health care. In Proceedings of the 7th International Symposium on Medical Information and Communication Technology. Tokyo, Japan: IEEE, 2013.

[32] C. Liu, C. Yang, X. Zhang, and J. Chen, "External integrity verification for outsourced big data in cloud and IoT. A big picture," Future Generation Computer Systems, vol. 49, pp. 58-67, August 2015.

[33] Y. Ma, J. Rao, W. Hu, X. Meng, X. Han, Y. Zhang, and C. Liu, "An efficient index for massive IoT data in cloud environment," [21st International Conference on Information and Knowledge Management]. Maui, Hawaii: ACM, 2012.

[34] C. Dores, L. P. Reis, and N. V. Lopes, "Internet of Things and cloud computing," [Iberian Conference on Information Systems \& Technologies]. Barcelona, Spain: IEEE, 2014. 